\title{
Implementing a protocol to prevent incisional hernia in high-risk patients: a mesh is a powerful tool
}

\author{
J. A. Pereira-Rodríguez ${ }^{1,2}$ (D) S. Amador-Gil ${ }^{3} \cdot$ A. Bravo-Salva $^{1,2} \cdot$ B. Montcusí-Ventura $^{1} \cdot$ J. Sancho-Insenser ${ }^{1,3}$. \\ M. Pera-Román ${ }^{1,3} \cdot$ M. López-Cano ${ }^{3,4}$
}

Received: 12 August 2021 / Accepted: 17 October 2021 / Published online: 1 November 2021

(c) The Author(s) 2021

\begin{abstract}
Purpose The small bites (SB) technique for closure of elective midline laparotomies (EMLs) and a prophylactic mesh (PM) in high-risk patients are suggested by the guidelines to prevent incisional hernias (IHs) and fascial dehiscence (FD). Our aim was to implement a protocol combining both the techniques and to analyze its outcomes.

Methods Prospective data of all EMLs were collected for 2 years. Results were analyzed at 1 month and during follow-up. The incidence of $\mathrm{HI}$ and FD was compared by groups $(\mathrm{M}=\mathrm{Mesh} v s . \mathrm{S}=$ suture $)$ and by subgroups depending on using SB. Results A lower number of FD appeared in the M group (OR 0.0692; 95\% CI 0.008-0.56; $P=0.01$ ) in 197 operations. After a mean follow-up of 29.23 months $(N=163$; min. 6 months), with a lower frequency of IH in M group (OR 0.769; 95\% CI 0.65-0.91; $P<0.0001)$. (33) The observed differences persisted after a propensity matching score: FD (OR 0.355; 95\% CI $0.255-0.494 ; P<0.0001)$ and IH (OR 0.394; 95\% CI 0.24-0.61; $P<0.0001)$. On comparing suturing techniques by subgroups, both mesh subgroups had better outcomes. PM was the main factor related to the reduction of IH (HR 11.794; 95\% CI 4.29-32.39; $P<0.0001)$.

Conclusion Following the protocol using PM and SB showed a lower rate of FD and HI. A PM is safe and effective for the prevention of both HI and FD after MLE, regardless of the closure technique used.
\end{abstract}

Keywords Abdominal wall closure $\cdot$ Laparotomy closure $\cdot$ Incisional hernia $\cdot$ Small bites $\cdot$ Short stitch $\cdot$ Prophylactic mesh

\section{Introduction}

The beneficial effect for the prevention of incisional hernia (IH) of closing a midline laparotomy with a running suture at a suture length/wound length ratio (SL/WL) of at least 4:1 $[1,2]$ has been recognized in several randomized controlled trials $[3,4]$. The recommendations of societies dedicated to abdominal wall surgery [2] and several comparative studies

J. A. Pereira-Rodríguez

jpereira@parcdesalutmar.cat

1 Department of General and Digestive Surgery, Hospital, Universitario del Mar. Parc de Salut Mar, Barcelona, Spain

2 Department of Experimental and Health Sciences, Universitat Pompeu Fabra, Barcelona, Spain

3 Department of Surgery and Morphological Sciences, Parc de Salut Mar, Hospital del Mar, Universitat Autónoma de Barcelona, Passeig Maritim 25-29, 08003 Barcelona, Spain

4 Department of General and Digestive Surgery, Hospital Valle de Hebrón, Barcelona, Spain
[3-7] propose combining a high SL/WL ratio with a "small bites" technique (SB) $[3,4]$, and the use of a prophylactic mesh (PM) [5-7] in high-risk patients. However, both measures have not been widely implemented [8-10], particularly those with high BMI, previous hernia repair, emergency surgery and contaminated/dirty surgery.

There are several reasons for explaining this reluctance, the main ones being that the SB technique has not been satisfactorily studied in high-risk patients, and the potential complications related to PM [8-10].

In a previous study in low-risk patients [11], application of a protocolized closure of the abdominal wall using the SB technique was difficult; however, it was superior in terms of prevention of IH.

We hypothesize that the combination of the SB technique with a PM for closure of the abdominal wall after midline laparotomy reduces the incidence of IH and fascial dehiscence (FD) in high-risk patients.

The main objective of this study is to implement a protocol combining the closure with SB associated with a 
suprafascial (onlay) PM in elective median laparotomy (EML) in high-risk patients and to evaluate its effectiveness for the prevention of complications related to abdominal wall closure.

\section{Methods}

We did a prospective, single-centre, observational study by means The Abdominal Wall Closure Update Hospital Program (PHACPA acronym in Spanish), which is an initiative included in the framework for improving the quality of health and patient care in a University Hospital (IMASQUAL) in Spain.

This program includes changes in the surgical technique of laparotomy closure, unifying the suture material type (polydioxanone 2/0 USP, HR 26 Monoplus ${ }^{\circledR}$, B. Braun. Melsungen, Germany), and using the SB technique and introducing the measurement and systematic documentation of the SL/WL ratio. For that purpose, training actions, which have been previously described, [11] were carried out, and all surgical specialties that perform EML were involved (General and Digestive Surgery, Gynecology, Urology, Vascular). In addition, the use of PM in high-risk patients was emphasised, following updated guidelines [2].

A polyvinylidene (PVDF) mesh (Cicat ${ }^{\circledR}$, Dynamesh, Aachen, Germany) in an onlay position, adjusted to the size of the incision with an overlap between 3 and $5 \mathrm{~cm}$, and fixed with a $2 / 0$ polypropylene running suture (Prolene ${ }^{\circledR}$, Johnson and Johnson, NYSE, USA) was used for prophylaxis.

All EMLs in patients with high risk of IH were prospectively included between July 2016 and July 2018. EMLs performed for extraction site or for hand assistance in patients undergoing laparoscopic surgery were also included. The inclusion criteria for the analysis were a minimum of two risk factors for IH [12-14]: age older than 70 years; body mass index (BMI) greater than $30 \mathrm{~kg} / \mathrm{m}^{2}$; and history of chronic obstructive pulmonary disease (COPD), abdominal aortic aneurysm (AAA), immunosuppression, malnutrition (albumin $<3 \mathrm{~g} / \mathrm{dL}$ ), chronic renal failure (CRF) (creatinine $>1.5 \mathrm{mg} / \mathrm{dL}$ ), operation for cancer, diabetes mellitus (DM), and smoking. Patients with a previous mesh or hernia present during surgery were excluded.

A common database for all laparotomies (PHACPA study) was designed to collect data of the patient's characteristics, pathology, operations, surgical wound classification according to Center of Diseases Control (CDC) [15], technique of abdominal wall closure, discharge, complications in the first 30 days classified by the Clavien-Dindo Grade [16] and specifically those of the wound (SSO, SSI, seroma, hematoma, fascial dehiscence and incisional hernia), and follow-up at 1 month, 6 months, 1 year, and thereafter. Data were collected prospectively on a data base designed with FileMaker Pro 15 (Claris International Inc) review of the report and medical records. Clinical follow-up was performed by the surgeon and/or oncologist with physical examination and abdominal CT scan when deemed necessary, data were collected prospectively for the data manager in each appointment. IH was defined according to the description of the EHS [17]: "Any abdominal wall gap with or without a bulge in the area of a postoperative scar perceptible or palpable by clinical examination or imaging". In patients with persistent purulent discharge, explantation of the mesh would be considered. Patients who presented with FD were operated using abdominal wall closure and suprafascial mesh placement.

For the analysis, patients were divided into two groups based on the use of PM (M Group) or not (S Group), and they were further divided into subgroups based on the technique of abdominal wall closure. Abdominal wall closure was considered according to the SB technique when the surgical report included the suggested suture material, with the SB technique and the result of the SL/WL ratio. Patients who met these criteria were included in the mesh-small bites (MSB) subgroup if received a PM; the remaining patients were assigned to the suture-small bites (SSB) subgroup. Cases where a different suture material or a different suture gauge was used and/or the calculation of SL/WL ratio was absent were assigned to the mesh-non-small bites (MNSB) subgroup when a PM was used; if not, they were considered in the suture-non-small bites (SNSB) subgroup. The use of $\mathrm{SB}$ and PM was at the surgeon's discretion. The distribution of the groups and subgroups is shown in Fig. 1.

\section{Statistical analysis}

The data were exported to the SPSS 25.0 statistical package (IBM Inc. Rochester, MN, USA). Quantitative variables were expressed as mean \pm standard deviation (SD) and qualitative variables as proportions. To analyze the association between qualitative variables, the Chi-squared test or Fisher's test was used when necessary, as well as Student's $t$ test or the Mann-Whitney test was used for quantitative variables. Normal distribution of the quantitative variables was verified using the Kolmogorov-Smirnov test. Statistical significance was established with $P<0.05$. A Cox proportional hazards regression model was used to detect the risk factors related to IH.

To reduce the effect of confounding variables, a propensity matching score (PMS) was performed. Scores were estimated by logistic regression analysis, with the treatment strategy group (Mvs. S) as the dependent variable and age, sex, and previous hernia as independent variables. Matching was performed according to the "nearest neighbor" method using a 0.2-width caliper and at a $2: 1$ ratio. 
Fig. 1 Flowchart

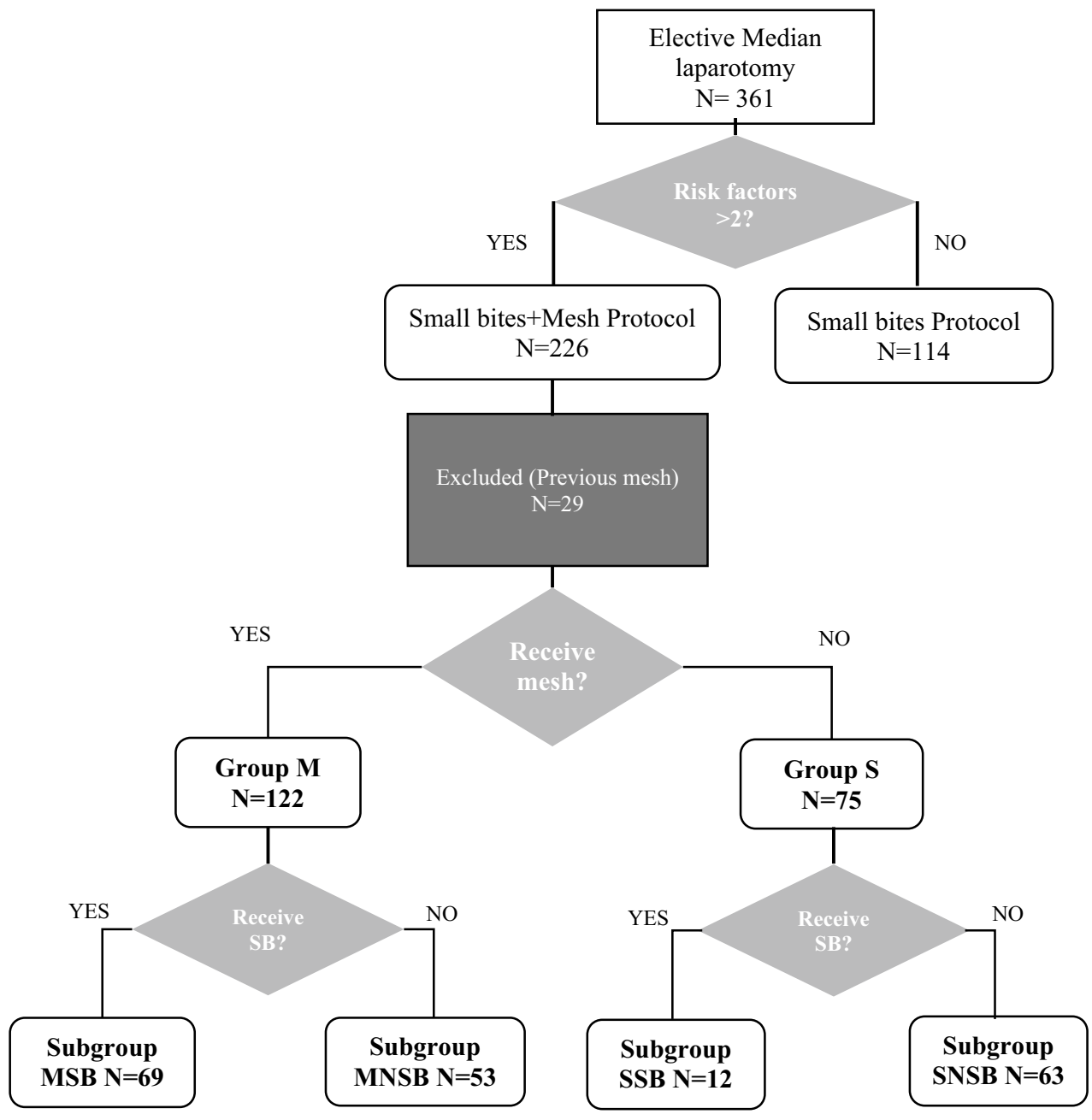

The Clinical Research Ethics Committee (CREC number 2016/6543/I) approval was obtained. Patients were informed, and data were processed according to Law 15/1999 on the Protection of Personal Data. The clinical trial protocol was registered with the NCT02658955 code (ClinicalTrials.gov).

\section{Results}

\section{Demographic data}

From July 2016 to July 2018, 226 patients from a University Hospital met the inclusion criteria and underwent surgery for LME among the 82 surgeons comprising part of the Surgical Specialties; 29 patients were excluded for carrying a previous mesh (Fig. 1), and 197 were considered valid for analysis.

Patient characteristics and comparison between the $\mathrm{M}$ and $\mathrm{S}$ groups are shown in Table 1. These groups showed statistically significant differences in terms of age, sex, and previous hernia repair, which were more frequent in the $\mathrm{S}$ group.

\section{Short-term postoperative comparison}

Table 2 presents the data of the operations and their results. Surgery was longer in $M$ group patients, who had a higher frequency of class III and IV wounds. The incision length was shorter in the $\mathrm{S}$ group, consistent with a higher proportion of laparotomies for surgical specimen removal (M Group 38 (31.1\%) vs S Group 44 (58.7\%); $P<0.0001)$, although the incision length was recorded in only 15 patients in group $\mathrm{S}$. The proportion of patients with surgical wound classification as type III and IV was higher in group M (15\% vs. 5.3\%; $P=0.038$ ), although the analysis of postoperative complication grade did not show significant differences between the two groups, except for a higher frequency of seromas in patients of the M group (OR 2.686; 95\% CI 1.10-6.54; $P=0.029$ ). In the postoperative period, only one patient $(0.9 \%)$ in group $M$ was diagnosed with FD, compared to eight $(11.9 \%)$ in group S (OR $0.0692 ; 95 \%$ CI $0.008-0.56 ; P=0.01)$; of which seven patients were not reported to be using SB (NSB). When comparing the cases after PMS (Table 3), a higher frequency of seromas persisted in the $\mathrm{M}$ group, although without statistical significance 
Table 1 Patient characteristics comparison

\begin{tabular}{lllll}
\hline & Total & Group M & Group S & \\
& $N=197$ & $N=122$ & $P$ \\
\hline Age, median (IQR) & $72.8(66.9-81.0)$ & $71.9(66.2-71.9)$ & $75.0(68.4-81.3)$ & 0.414 \\
Age $>70$ years, $N(\%)$ & $124(62.9)$ & $69(56.6)$ & $55(73.3)$ & 0.018 \\
Female sex, $N(\%)$ & $80(40.6)$ & $59(48.4)$ & $21(28.0)$ & 0.005 \\
ASA $\geq$ III, $N(\%)$ & $111(56.3)$ & $73(59.8)$ & $38(50.7)$ & 0.208 \\
BMI $\left(\mathrm{kg} / \mathrm{m}^{2}\right)$, media $N(\mathrm{IQR})$ & $27.3(24.2-30.3)$ & $26.5(23.9-30.0)$ & $27.9(24.4-30.5)$ & 0.326 \\
BMI $>30 \mathrm{~kg} / \mathrm{m}^{2}, N(\%)$ & $67(34.9)$ & $38(31.7)$ & $29(40.3)$ & 0.226 \\
Smoking, $N(\%)$ & $35(17.8)$ & $22(18.0)$ & $13(17.3)$ & 0.901 \\
DM, $N(\%)$ & $52(26.4)$ & $32(26.2)$ & $20(26.7)$ & 0.946 \\
COPD $N(\%)$ & $51(25.9)$ & $28(23.0)$ & $23(30.7)$ & 0.230 \\
CRF, $N(\%)$ & $20(10.2)$ & $13(10.7)$ & $7(9.3)$ & 0.765 \\
Cancer operation, $N(\%)$ & $168(85.3)$ & $101(82.8)$ & $67(89.3)$ & 0.208 \\
Previous laparotomy, $N(\%)$ & $39(19.8)$ & $29(23.8)$ & $10(13.3)$ & 0.074 \\
Previous hernia, $N(\%)$ & $38(19.3)$ & $16(13.1)$ & $22(29.3)$ & 0.005 \\
Immunosuppression, $N(\%)$ & $16(8.1)$ & $12(9.8)$ & $4(5.3)$ & 0.261 \\
AAA, $N(\%)$ & $6(3.0)$ & $5(4.1)$ & $1(1.3)$ & 0.273 \\
\hline
\end{tabular}

$S D$ standard deviation; IQR interquartile range; ASA American Society of Anesthesiologists; BMI body mass index; $D M$ diabetes mellitus; $C O P D$ chronic obstructive pulmonary disease; $C R F$ chronic renal failure; $A A A$ abdominal aortic aneurysm
(OR 1.818; CI 0.86-3.84; $P=0.084$ ), and a significant difference in FD was maintained in favor of the M group (OR 0.355; 95\% CI 0.255-0.494; $P<0.0001)$.

\section{Long-term postoperative comparison}

A total of 163 patients completed a mean follow-up of 29.23 months (SD 12.5) (Group M 28.66 vs. Group S 25.62; $P=0.272$ ), with a statistically significant lower frequency of $\mathrm{IH}$ in $\mathrm{M}$ group patients (M group 7 (7.2\%) vs $\mathrm{S}$ group 13 (29.5\%); OR 0.769; 95\% CI 0.65-0.91; $P<0.0001)$. Similar results were obtained by PMS (OR 0.394; 95\% CI 0.24-0.61; $P<0.0001$ ) (Table 3). No patients of $\mathrm{M}$ group presented chronic mesh infection or requires mesh explantation during follow-up.

Comparing by the subgroups, when a PM was used (Subgroups MSB and MNSB), a more favorable yield was obtained in the incidences of IH and FD regardless of the suturing technique (Table 3 and Fig. 2a, b). Analyzing suturing technique independently, a higher incidence of IH and FD was observed in patients where SB was not performed (MNSB and SNSB) (Table 3). Cox multivariate analysis revealed the use of a PM as the only factor related to prevention of IH (HR 11.794; 95\% CI 4.29-32.39; $P<0.0001)$ (Fig. 3).

\section{Discussion}

The results of our study show that adequate fulfillment for closure of EMLs using SB and a PM in high-risk patients correlates with a lower frequency of IH and FD. The main related factor was the use of a PM, independent of the technique for EML closure.

Thus far, studies about EML closure have shown better results when using the SB technique, but they were carried out in non-selected patients $[3,4,11]$. In our study, isolated use of SB, although in a small number of patients, presented a similar frequency of IH than in those treated with NSB, indicating that this group of patients could not benefit as much from this technique as low-risk patients. However, the absence of this data on the operative report was classified as non-compliance with the protocol (NSB), although it could be that it had been correctly applied, a fact that could influence the lack of significance in our sample. We think it is necessary to emphasize to surgeons the importance of measuring and reflecting this data to insure conclusions. Therefore, more research on SB technique in high-risk patients is needed.

For high-risk IH patients, the European Hernia Society (EHS) guidelines [2] suggest the use of a PM, and, in a recent randomized study, the onlay position presented greater advantages [18]. Nevertheless, its use has not become widespread for the following reasons: increased costs, possibility of increased complications related to the wound, as well as concerns about legal consequences derived from the use of a prosthesis [9]. As reported previously [7, 18-20], our study shows that using a PM is safe also for contaminated surgery; it is indeed associated with a higher frequency of seromas, but it also implies a clear decrease in the frequency of both IH and FD; thus, in our opinion, the benefit justifies the risk of a minor complication, such as seroma. 
Table 2 Intraoperative characteristics and postoperative complications comparison

\begin{tabular}{|c|c|c|c|c|}
\hline & $\begin{array}{l}\text { Total } \\
N=197\end{array}$ & $\begin{array}{l}\text { Group M } \\
N=122\end{array}$ & $\begin{array}{l}\text { Group S } \\
N=75\end{array}$ & $P$ \\
\hline Operative time (minutes), mean (SD) & $226.9(94.3)$ & $238.6(91.1)$ & $207.8(96.8)$ & 0.026 \\
\hline Extraction site midline laparotomy, $N(\%)$ & $82(41.6)$ & $38(31.1)$ & $44(58.7)$ & $<0.0001$ \\
\hline SL/WL ratio, median $(\mathrm{IQR})^{\mathrm{a}}$ & $4.75(4.0-6.1)$ & $4.80(4.0-6.1)$ & $4.16(4.0-6.8)$ & 0.783 \\
\hline Length of hospital stay (days), median (IQR) & $7.0(5.0-13.0)$ & $7.0(5.0-13.0)$ & $6.0(4.0-13.0)$ & 0.319 \\
\hline \multicolumn{5}{|l|}{ Surgical wound classification } \\
\hline Grade I & $61(30.9)$ & $24(19.7)$ & $37(49.3)$ & \\
\hline Grade II & $114(57.9)$ & $80(65.6)$ & $34(45.3)$ & \\
\hline Grade III & $21(10.7)$ & $17(13.9)$ & $4(5.3)$ & \\
\hline Grade IV & $1(0.5)$ & $1(0.8)$ & $0(0.0)$ & \\
\hline Grade III-IV, $N(\%)$ & $22(11.3)$ & $18(15.0)$ & $4(5.3)$ & 0.038 \\
\hline \multicolumn{5}{|l|}{ Specialties } \\
\hline General and digestive surgery, $N(\%)$ & $125(63.5)$ & $97(77.6)$ & $28(22.4)$ & \\
\hline Gynecology, $N(\%)$ & $18(9.1)$ & $14(77.8)$ & $4(22.2)$ & \\
\hline Urology, $N(\%)$ & $46(23.4)$ & $3(6.5)$ & $43(93.5)$ & \\
\hline Vascular, $N(\%)$ & $8(4.1)$ & $8(100)$ & & \\
\hline \multicolumn{5}{|l|}{ Complications grade } \\
\hline Grade $0, N(\%)$ & $48(24.4)$ & $27(22.1)$ & $21(28.0)$ & 0.351 \\
\hline Grade I, $N(\%)$ & $37(18.8)$ & $26(21.3)$ & $11(14.7)$ & \\
\hline Grade II, $N(\%)$ & $75(38.1)$ & $49(40.2)$ & $26(34.7)$ & \\
\hline Grade IIIa, $N(\%)$ & $9(4.6)$ & $7(5.7)$ & $2(2.7)$ & \\
\hline Grade IIIb, $N(\%)$ & $22(11.2)$ & $12(9.8)$ & $10(13.3)$ & \\
\hline Grade IV, $N(\%)$ & $4(2.0)$ & $1(0.8)$ & $3(4.0)$ & \\
\hline Grade V, $N(\%)$ & $2(1.0)$ & $0(0.0)$ & $2(2.7)$ & 0.070 \\
\hline \multicolumn{5}{|l|}{ Wound complications } \\
\hline $\mathrm{SSO}, N(\%)$ & $77(39.1)$ & $50(41.0)$ & $27(36.0)$ & 0.486 \\
\hline SSI, $N(\%)$ & $44(22.3)$ & $27(22.1)$ & $17(22.7)$ & 0.930 \\
\hline Superficial SSI, $N(\%)$ & $24(54.5)$ & $14(51.9)$ & $10(58.8)$ & \\
\hline Deep SSI, $N(\%)$ & $7(15.9)$ & $5(18.5)$ & $2(11.8)$ & \\
\hline Organ space, $N(\%)$ & $13(29.5)$ & $8(29.6)$ & $5(29.4)$ & \\
\hline Seroma, $N(\%)$ & $33(16.8)$ & $26(21.3)$ & $7(9.3)$ & 0.029 \\
\hline Hematoma, $N(\%)$ & $12(6.1)$ & $6(4.9)$ & $6(8.0)$ & 0.380 \\
\hline Fascial dehiscence, $N=181(\%)$ & $9(5.0)$ & $1(0.9)$ & $8(11.3)$ & 0.002 \\
\hline Subgroups SB, $N=77(\%)$ & $2(2.6)$ & $1(1.6)$ & $1(8.3)$ & 0.30 \\
\hline Subgroups NSB, $N=104(\%)$ & $7(6.7)$ & $0(0.0)$ & $7(11.9)$ & 0.016 \\
\hline
\end{tabular}

$S D$ Standard deviation; IQR Interquartile range; SSO Surgical site occurrence; SSI Surgical site infection

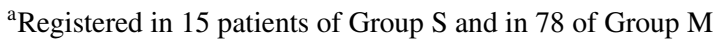

FD itself is a serious problem, associated with severe complications and high mortality [21, 23]. Its prevention justifies the use of a PM in these fragile patients who have associated comorbidities to avoid reoperations and, at the same time, prevent a future IH and its impact on the patient's quality of life and costs. Our data confirm the results of previous studies, in which a PM successfully prevented the appearance of FD [24].

One detail to highlight in our study is that, despite previous teaching work and seminars of the protocol in all participants, a considerable number of surgeons did not fully use it. Our results shows a FD rate of $11.9 \%$ and IH rate of $29.5 \%$ if the protocol is not followed and this can be improved to $1.6 \% \mathrm{FD}$ and $9.1 \% \mathrm{IH}$ observed if the protocol is performed correctly. These data are similar to those reported in previous studies [5-7]. Jairam et al. [18] observed an IH rate in patients with primary suture of $30 \%$ versus $13 \%$ and $18 \%$ observed in patients with onlay and sublay mesh, respectively. Borab et al. [6] described an $85 \%$ reduction in the rate of IH with mesh placement and Garcia-Urena et al. [7] documented a rate of $31.5 \%$ in the non-mesh control group and $11.3 \%$ in the mesh study group.

Only 35\% of patients received both PM and SB closure. This result is concerning and highlights the need for 
Table 3 PSM analysis comparison

\begin{tabular}{|c|c|c|c|c|}
\hline & $\begin{array}{l}\text { Total } \\
N=173\end{array}$ & $\begin{array}{l}\text { Group M } \\
N=115\end{array}$ & $\begin{array}{l}\text { Group S } \\
N=58\end{array}$ & $P$ \\
\hline Operative time (minutes), mean (SD) & $229.5(92.9)$ & $238.7(91.7)$ & $212.1(93.2)$ & 0.811 \\
\hline Extraction site midline laparotomy, $N(\%)$ & $70(40.5)$ & $34(58.6)$ & $36(31.3)$ & 0.001 \\
\hline SL/WL ratio, median $(\mathrm{IQR})^{\mathrm{a}}$ & $4.80(4.0-6.9)$ & $4.75(4.0-6.2)$ & $4.80(4.0-7.6)$ & 0.798 \\
\hline Length of hospital stay (days), median (IQR) & $7.0(5.0-12.0)$ & $7.0(5.0-12.0)$ & $6.5(4.0-12.2)$ & 0.593 \\
\hline \multicolumn{5}{|l|}{ Surgical wound classification } \\
\hline Grade I & $51(29.5)$ & $22(19.1)$ & $29(50.0)$ & \\
\hline Grade II & $103(59.5)$ & $76(66.1)$ & $27(46.5)$ & \\
\hline Grade III & $18(10.4)$ & $16(13.9)$ & $2(3.5)$ & \\
\hline Grade IV & $1(0.6)$ & $1(0.9)$ & $0(0.0)$ & \\
\hline Surgical wound classification III-IV, $N(\%)$ & $19(11.1)$ & $17(15.0)$ & $2(3.4)$ & 0.022 \\
\hline \multicolumn{5}{|l|}{ Specialties } \\
\hline General and Digestive Surgery, $N(\%)$ & $114(65.9)$ & $90(78.9)$ & $24(21.1)$ & \\
\hline Gynecology, $N(\%)$ & $18(10.4)$ & $14(77.8)$ & $4(22.2)$ & \\
\hline Urology, $N(\%)$ & $33(19.1)$ & $3(9.1)$ & $30(90.9)$ & \\
\hline Vascular, $N(\%)$ & $8(4.6)$ & $8(100.0)$ & $0(0.0)$ & \\
\hline \multicolumn{5}{|l|}{ Complications Grade } \\
\hline Grade $0, N(\%)$ & $44(25.4)$ & $26(22.6)$ & $18(31.0)$ & \\
\hline Grade I, $N(\%)$ & $33(19.1)$ & $25(21.7)$ & $8(13.8)$ & \\
\hline Grade II, $N(\%)$ & $63(36.4)$ & $46(40.0)$ & $17(29.3)$ & \\
\hline Grade IIIa, $N(\%)$ & $7(4.0)$ & $5(4.3)$ & $2(3.4)$ & \\
\hline Grade IIIb, $N(\%)$ & $21(12.1)$ & $12(10.4)$ & $9(15.5)$ & \\
\hline Grade IV, $N(\%)$ & $3(1.7)$ & $1(0.9)$ & $2(3.4)$ & \\
\hline Grade V, $N(\%)$ & $2(1.2)$ & $0(0.0)$ & $2(3.4)$ & \\
\hline Overall complications & $129(74.6)$ & $89(77.4)$ & $40(69.0)$ & 0.230 \\
\hline \multicolumn{5}{|l|}{ Wound complications } \\
\hline $\mathrm{SSO}, N(\%)$ & $67(38.7)$ & $45(39.1)$ & $22(37.9)$ & 0.878 \\
\hline SSI, $N(\%)$ & $38(22.0)$ & $24(20.9)$ & $14(24.1)$ & 0.624 \\
\hline Superficial SSI, $N(\%)$ & $21(55.3)$ & $1458.3)$ & $7(50.0)$ & \\
\hline Deep SSI, $N(\%)$ & $6(15.8)$ & $4(16.7)$ & $2(14.3)$ & \\
\hline Organ space, $N(\%)$ & $11(28.9)$ & $6(25.0)$ & $5(35.7)$ & \\
\hline Seroma, $N(\%)$ & $30(17.3)$ & $24(20.9)$ & $6(10.3)$ & 0.084 \\
\hline Hematoma, $N(\%)$ & $9(5.2)$ & $4(3.5)$ & $5(8.6)$ & 0.150 \\
\hline Fascial dehiscence, $N=158(\%)$ & $9(5.7)$ & $1(1.0)$ & $8(14.5)$ & $<0.0001$ \\
\hline Subgroups SB, $N=71(\%)$ & $2(2.8)$ & $1(1.7)$ & $1(10.0)$ & 0.27 \\
\hline Subgroups NSB, $N=87(\%)$ & $7(8.0)$ & $0(0.0)$ & $7(15.6)$ & 0.012 \\
\hline Incisional hernia, $N=141(\%)$ & $20(14.2)$ & $7(7.2)$ & $13(29.5)$ & $<0.0001$ \\
\hline Subgroups SB, $N=65(\%)$ & $8(12.3)$ & $5(9.1)$ & $3(37.5)$ & 0.057 \\
\hline Subgroups NSB $N=76(\%)$ & $12(15.8)$ & $2(4.8)$ & $10(27.8)$ & 0.009 \\
\hline
\end{tabular}

$S D$ standard deviation; IQR interquartile range; ASA American Society of Anesthesiologists; BMI body mass index; $D M$ diabetes mellitus; $C O P D$ chronic obstructive pulmonary disease; $C R F$ chronic renal failure; $A A A$ abdominal aortic aneurysm

${ }^{\text {a }}$ Registered in 12 patients of Group S and in 45 of Group M encouraging good compliance of protocols and information to surgeons and surgical departments the results of their application. Of particular concern is the low use of SB, and in our study has precluded properly comparing both suturing techniques due to lack of sample. This finding is not exclusive of our study as previously reported [25] only $42 \%$ of surgeons followed the EHS guidelines on abdominal wall closure. It is likely that the lack of compliance with SB is related with the lack of measurement of the SL/WL or with lack of confidence on using a $2 / 0$ suture more than a deficiency of knowledge.

It is also striking that in a higher number of procedures, a PM was used (61.9\%), which suggests a lower trust by surgeons in the SB technique and a greater penetration as 
Fig. 2 Incisional hernia (a) and Fascial dehiscence (b) analysis by groups and subgroups. Using Group $\mathrm{S}$ as reference
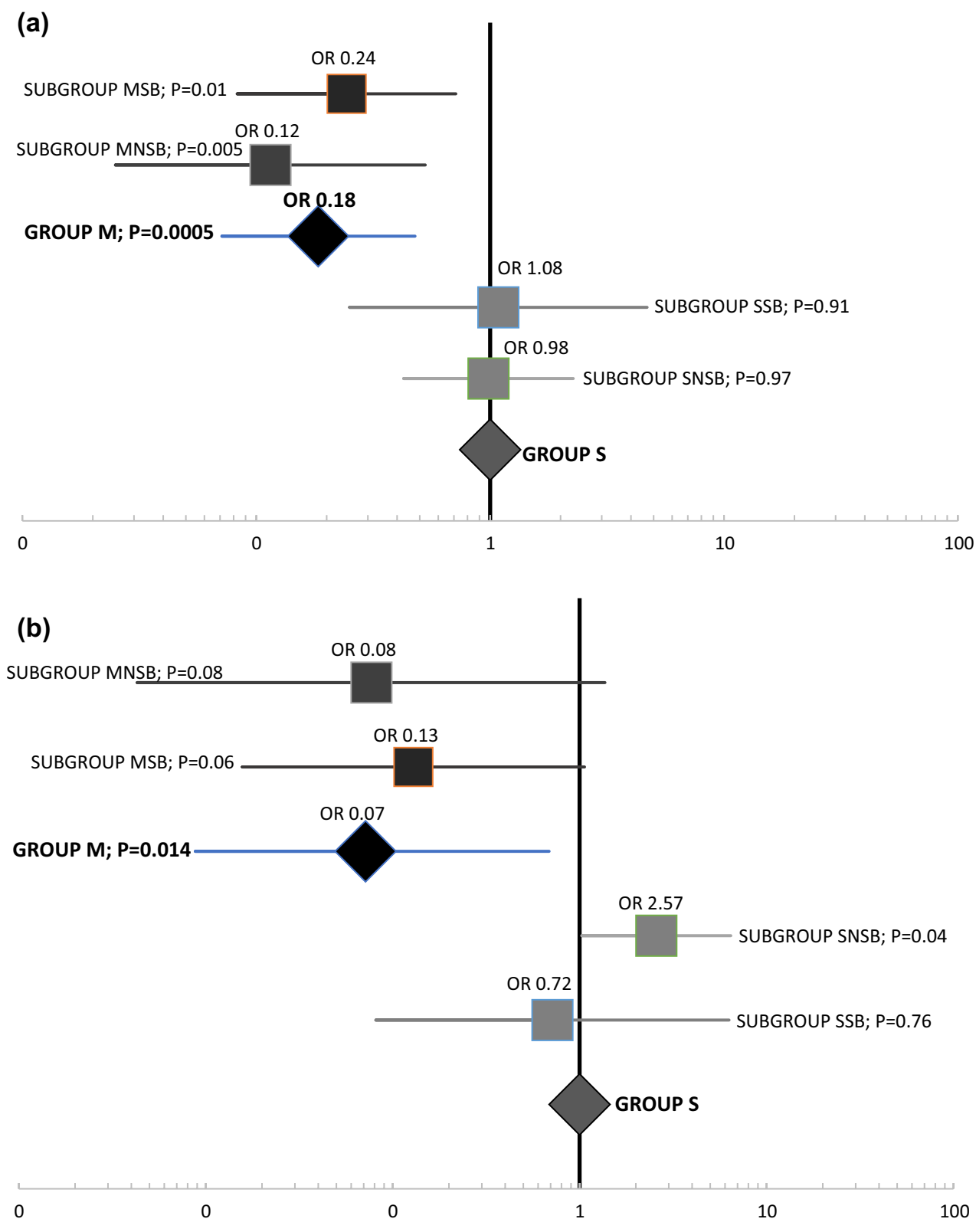

a recommendation for the use of PM in these high-risk patients. It was probably because PM has a longer scientific background [7, 18-27].

In the same line, a higher proportion of incisions in $\mathrm{S}$ group patients $(58.7 \%)$ were related to small/mid-size laparotomies for removal of a specimen or assistance during laparoscopy. Probably, surgeons, when dealing with small incisions, are likely to underestimate the risk of IH and FD. Once analyzed separately, these patients had a higher frequency of FD when not receiving a mesh (M group $1.4 \%$ vs. S group $22.2 \% ; P<0.0001)$, and statistical significance was not reached in IH (M group $9.1 \%$ vs. S group $21.1 \% ; P=0.154)$ probably due to the sample size.
These data confirm that incisions for assistance or specimen extraction in high-risk patients have a similar risk of IH and FD as those with open surgery; therefore, the size of the incision does not seem to be related to $\mathrm{IH}$, as noted previously [27-29].

The main strengths of our study are that it has been carried out prospectively, studying the application in a real setting of a unified technique through learning. The mean follow-up over 2 years ensures there have been no chronic complications related to the use of meshes. Finally, the use of PMS has allowed avoiding biases, which were produced by lack of randomization of patient groups. 
Fig. 3 Incisional hernia during follow-up by use of mesh. HR 11.794; CI 4.29-32.39; $P<0.0001$

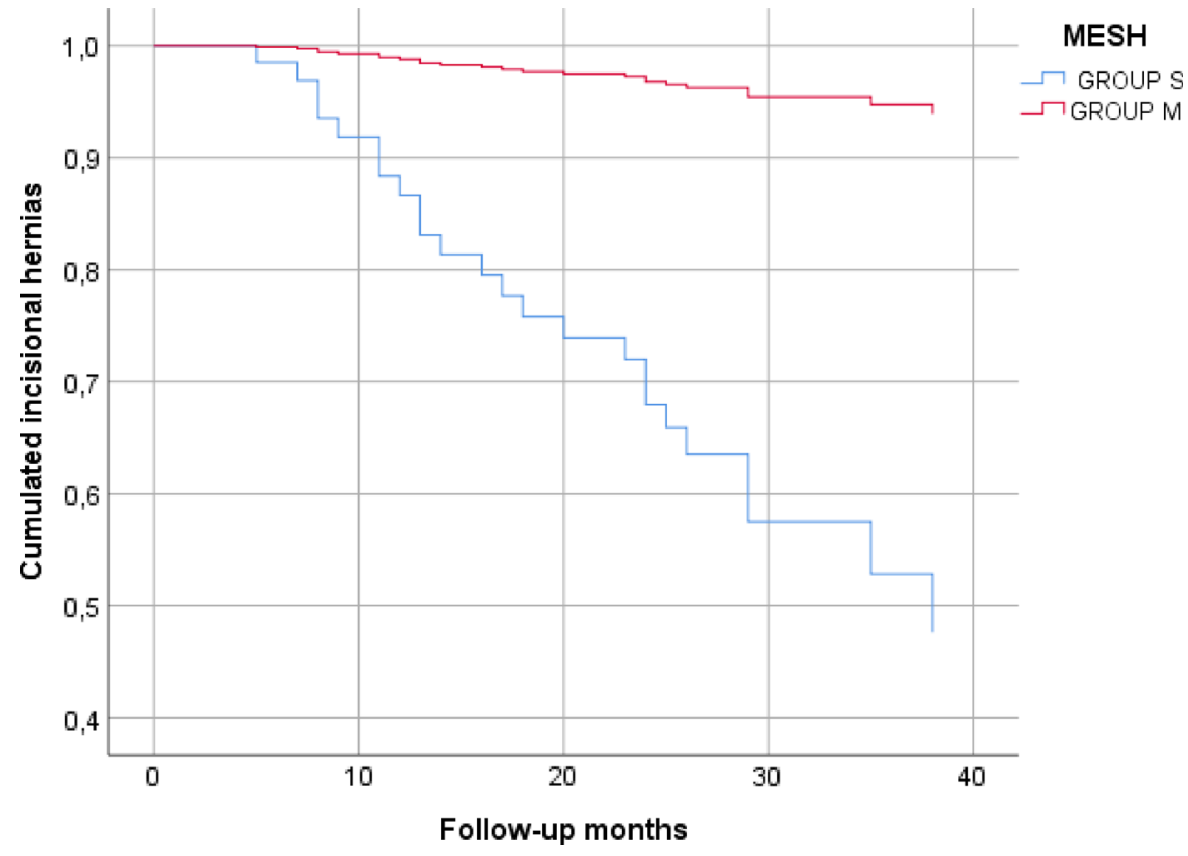

\begin{tabular}{|llllll|}
\hline GROUP M & 122 & 100 & 96 & 73 & 49 \\
GROUP S & 75 & 57 & 53 & 39 & 19 \\
\hline
\end{tabular}

There are some weaknesses of our study. The main is related to the decrease in sample size when analyzing subgroups. Also, their characteristics, although it is prospective research, the lack of greater compliance of the protocol makes it difficult to derive definitive conclusions from the comparison of the closure techniques. The suturing technique as well as the use of mesh was at the choice of the surgeon in responsible. During the period of study, all the participating services were informed about postoperative results and the percentage of fulfillment of the protocol every 6 months encouraging them to improve. The specialties of General Surgery, Gynecology and Vascular Surgery were more aware of the use of PM and had better completion of the protocol, while Urology still seems to lack confidence in its use and had worst results. We believe that the correct closure of the abdominal wall should be known and performed equally in all departments that perform LME, given that the wound complications entail a considerable morbimortality for patients, which we believe can be improved.

In conclusion, in patients with risk factors for IHs who are undergoing EML, following the protocol using PM and SB showed a lower rate of FD and HI. A PM is the appears to be a powerful tool for prevention of both IH and FD, regardless the closure technique used.

Acknowledgements We thank Parc de Salut Mar for funding, and Marta Gimeno López, Elia Alonso Simón, and Lucía Fernández Yagüe for their help in collecting the data. We also thank LetPub (www.letpub.com) for its linguistic assistance during the preparation of this manuscript.
Funding Open Access funding provided thanks to the CRUE-CSIC agreement with Springer Nature. The investigation was funded by "Projectes de Millora de la Qualitat del Parc de Salut Mar," November 2015.

Data availability The datasets analyzed during the current study are available by request.

\section{Declarations}

Conflict of interest The authors declare that they have no competing interests.

Ethical approval The Clinical Research Ethics Committee (CREC number 2016/6543/I) approval was obtained.

Human and animal rights All procedures involving human participants were in accordance with ethical standards of the institution and with the 1964 Helsinki Declaration. Data were processed according to Law $15 / 1999$ on the Protection of Personal Data.

Informed consent Informed consent was obtained from all individual participants included in the study.

Consent for publication All authors have approved the manuscript and meet the requirements for authorship.

Open Access This article is licensed under a Creative Commons Attribution 4.0 International License, which permits use, sharing, adaptation, distribution and reproduction in any medium or format, as long as you give appropriate credit to the original author(s) and the source, provide a link to the Creative Commons licence, and indicate if changes were made. The images or other third party material in this article are included in the article's Creative Commons licence, unless indicated otherwise in a credit line to the material. If material is not included in 
the article's Creative Commons licence and your intended use is not permitted by statutory regulation or exceeds the permitted use, you will need to obtain permission directly from the copyright holder. To view a copy of this licence, visit http://creativecommons.org/licenses/by/4.0/.

\section{References}

1. Jenkins TP (1976) The burst abdominal wound: a mechanical approach. Br J Surg 63(11):873-876. https://doi.org/10.1002/ bjs. 1800631110

2. Muysoms FE, Antoniou SA, Bury K, Campanelli G, Conze J, Cuccurullo D et al (2015) European Hernia Society guidelines on the closure of abdominal wall incisions. Hernia 19(1):1-24

3. Deerenberg EB, Harlaar JJ, Steyerberg EW, Lont HE, van Doorn HC, Heisterkamp J et al (2015) Small bites versus large bites for closure of abdominal midline incisions (STITCH): a double-blind, multicentre, randomised controlled trial. Lancet 386(10000):1254-1260

4. Millbourn D, Cengiz Y, Israelsson LA (2009) Effect of stitch length on wound complications after closure of midline incisions: a randomized controlled trial. Arch Surg 144(11):1056-1059. https://doi.org/10.1001/archsurg.2009.189

5. Jairam AP, Timmermans L, Eker HH, Pierik REGJM, van Klaveren D, Steyerberg EW, Timman R, van der Ham AC, Dawson I, Charbon JA, Schuhmacher C, Mihaljevic A, Izbicki JR, Fikatas P, Knebel P, Fortelny RH, Kleinrensink GJ, Lange JF, Jeekel HJ, PRIMA Trialist Group (2017) Prevention of incisional hernia with prophylactic onlay and sublay mesh reinforcement versus primary suture only in midline laparotomies (PRIMA): 2-year follow-up of a multicentre, double-blind, randomised controlled trial. Lancet 390(10094):567-576

6. Borab ZM, Shakir S, Lanni MA et al (2017) Does prophylactic mesh placement in elective, midline laparotomy reduce the incidence of incisional hernia? A systematic review and metaanalysis. Surgery 161(4):1149-1163. https://doi.org/10.1016/j. surg.2016.09.036

7. García-Ureña MÁ, López-Monclús J, Hernando LA et al (2015) Randomized controlled trial of the use of a large-pore polypropylene mesh to prevent incisional hernia in colorectal surgery. Ann Surg 261(5):876-881. https://doi.org/10.1097/SLA.0000000000 001116

8. Yheulon C, Davis SS Jr (2019) Adopting the STITCH Trial: crossing the chasm from publication to practice. JAMA Surg 154(12):1087-1088. https://doi.org/10.1001/jamasurg.2019.3358

9. Bloemen A, De Kleijn RJCMF, Van Steensel S, Aarts F, Schreinemacher MHF, Bouvy ND (2019) Laparotomy closure techniques: Do surgeons follow the latest guidelines? Results of a questionnaire. Int J Surg 71:110-116. https://doi.org/10.1016/j.jisu.2019. 09.024

10. Fischer JP, Harris HW, López-Cano M, Hope WW (2019) Hernia prevention: practice patterns and surgeons' attitudes about abdominal wall closure and the use of prophylactic mesh. Hernia 23(2):329-334. https://doi.org/10.1007/s10029-019-01894-z

11. Pereira Rodríguez JA, Amador-Gil S, Bravo-Salva A et al (2021) Small bites technique for midline laparotomy closure: from theory to practice: Still a long way to go. Surgery 170:140-145. https:// doi.org/10.1016/j.surg.2020.12.007

12. Itatsu K, Yokoyama Y, Sugawara G, Kubota H et al (2014) Incidence of and risk factors for incisional hernia after abdominal surgery. Br J Surg 101(11):1439-1447
13. Sørensen LT, Kallehave F, Wille-Jørgensen P, Kjærgaard J, Nørgaard L, Jørgensen T (2005) Risk factors for tissue and wound complications in gastrointestinal surgery. Ann Surg 241:654-658

14. Van Ramshorst GH, Nieuwenhuizen J, Hop WCJ et al (2010) Abdominal wound dehiscence in adults: development and validation of a risk model. World J Surg 34:20. https://doi.org/10. 1007/s00268-009-0277-y

15. Surgical Site Infection (SSI) Event: Center for Disease Control. 2010. http://www.cdc.gov/nhsn/PDFs/pscManual/9pscSSIcurrent. pdf?agree $=$ yes\&next=Accept. Updated January 2021. Accessed March 28, 2021

16. Dindo D, Demartines NCP (2004) Classification of surgical complications. A new proposal with evaluation in a cohort of 6336 patients and results of a survey. Ann Surg 240:205-213

17. Kroese LF, Sneiders D, Kleinrensink GJ, Muysoms F, Lange JF (2018) Comparing different modalities for the diagnosis of incisional hernia: a systematic review. Hernia 22(2):229-242. https:// doi.org/10.1007/s10029-017-1725-5

18. Jairam AP, Timmermans L, Eker HH et al (2017) Prevention of incisional hernia with prophylactic onlay and sublay mesh reinforcement versus primary suture only in midline laparotomies (PRIMA): 2-year follow-up of a multicentre, double-blind, randomised controlled trial. Lancet 390(10094):567-576. https://doi. org/10.1016/S0140-6736(17)31332-6 ([published correction appears in Lancet. 2017 Aug 5;390(10094):554])

19. Caro-Tarrago A, Olona Casas C, Jimenez Salido A, Duque Guilera E, Moreno Fernandez F, Vicente Guillen V (2014) Prevention of incisional hernia in midline laparotomy with an onlay mesh: a randomized clinical trial. World J Surg 38(9):2223-2230. https:// doi.org/10.1007/s00268-014-2510-6

20. Muysoms FE, Detry O, Vierendeels T et al (2016) Prevention of incisional hernias by prophylactic mesh-augmented reinforcement of midline laparotomies for abdominal aortic aneurysm treatment: a randomized controlled trial. Ann Surg 263(4):638-645. https:// doi.org/10.1097/SLA.0000000000001369

21. Denys A, Monbailliu T, Allaeys M, Berrevoet F, van Ramshorst GH (2021) Management of abdominal wound dehiscence: update of the literature and meta-analysis. Hernia 25(2):449-462. https:// doi.org/10.1007/s10029-020-02294-4

22. López-Cano M, García-Alamino JM, Antoniou SA et al (2018) EHS clinical guidelines on the management of the abdominal wall in the context of the open or burst abdomen. Hernia 22(6):921939. https://doi.org/10.1007/s10029-018-1818-9

23. López-Cano M, Pereira JA, Armengol-Carrasco M (2013) "Acute postoperative open abdominal wall": Nosological concept and treatment implications. World J Gastrointest Surg 5(12):314-320. https://doi.org/10.4240/wjgs.v5.i12.314

24. Lima HVG, Rasslan R, Novo FCF et al (2020) Prevention of fascial dehiscence with onlay prophylactic mesh in emergency laparotomy: a randomized clinical trial. J Am Coll Surg 230(1):76-87. https://doi.org/10.1016/j.jamcollsurg.2019.09.010

25. de Vries HS, Verhaak T, van Boxtel TH et al (2020) Implementation of the small bites closure of abdominal midline incisions in clinical practice is correlated with a reduction in surgical site infections. Hernia 24(4):839-843. https://doi.org/10.1007/ s10029-019-01995-9

26. Ihedioha U (2008) Laparoscopic colorectal resection does not reduce incisional hernia rates when compared with open colorectal resection. Surg Endosc 22:689-692

27. Goodenough CJ, Ko TC, Kao LS et al (2015) Development and validation of a risk stratification score for ventral incisional hernia after abdominal surgery: hernia expectation rates in intraabdominal surgery (the HERNIA Project). J Am Coll Surg 220(4):405-413

28. Lee L, Mappin-Kasirer B, Sender Liberman A, Stein B, Charlebois P, Vassiliou M, Fried GM, Feldman LS (2012) High incidence of 
symptomatic incisional hernia after midline extraction in laparoscopic colon resection. Surg Endosc 26(11):3180-3185

29. Pereira JA, Pera M, López-Cano M et al (2019) Hernias at the extraction incision after laparoscopic colon and rectal resection: influence of incision location and use of prophylactic mesh. Cir Esp 97(1):20-26. https://doi.org/10.1016/j.ciresp.2018.08.002
Publisher's Note Springer Nature remains neutral with regard to jurisdictional claims in published maps and institutional affiliations. 